\title{
Authorship, institutional and citation metrics for publications on postmenopausal osteoporosis
}

\author{
M. H. Biglu • M. Ghavami • S. Biglu
}

Received: 21 July 2013 / Accepted: 10 December 2013 / Published online: 15 February 2014

(C) International Osteoporosis Foundation and National Osteoporosis Foundation 2014

\begin{abstract}
Introduction Osteoporosis is the most common metabolic bone condition that does not often become clinically clear until a fracture occurs. The objective of the current study was to analyze all publications whose titles included the term "postmenopausal osteoporosis" published during the past decade by journals indexed in the database of SCI-E.

Methods This paper analyzes two sets of data: in the first, all papers with "postmenopausal osteoporosis" in their titles indexed in the database of SCI-E in the period 2001-2011; the second, all papers published by Osteoporosis International that were indexed in SCI-E during 2001-2011. The Science of Science Tool was used to map the co-authorship networks of papers published by Osteoporosis International in 2007-2011. Only papers cited more than 100 times in the Web of Science were considered for mapping the co-authorship network.

Results A total number of 2,056 papers with "postmenopausal osteoporosis" in their titles were indexed in SCI-E between 2001 and 2011. The annual number of publications increased during the study period. The majority of publications came from Western Europe and North America. The number of papers published by authors based in Western Europe was about $75 \%$ greater than for North America.

Conclusion More papers on postmenopausal osteoporosis were published in Western Europe than in North America. The networks of co-authorship pointed to the strategic positions of highly cited authors from Western Europe. The top eight authors contributing the majority of papers were from Western Europe. Consequently Western Europe had greater impact than North America.
\end{abstract}

M. H. Biglu $(\bowtie) \cdot$ M. Ghavami $\cdot$ S. Biglu

Tabriz University of Medical Sciences,

Tabriz, Islamic Republic of Iran

e-mail: biglu@tbzmed.ac.ir
Keywords Menopausal women · Osteoporosis · Research activity $\cdot$ Scientometrics

\section{Introduction}

Osteoporosis is the most common metabolic bone condition and one which often is clinically silent until a fracture occurs. This has led to it being called the "silent illness." The bone fractures generally appear in the spine, wrist, and hip. Osteoporosis is a major public health problem that affects hundreds of millions of people worldwide, mainly postmenopausal women [1]. Menopause can increase a woman's risk of developing osteoporosis, a condition in which bones are weakened and may fracture easily. The decrease in the estrogen level that occurs around the time of menopause can lead to increased bone damage. It is estimated that the average woman loses up to $10 \%$ of her bone mass during the first 5 years after menopause [2]. Osteoporosis is responsible for over 1.5 million breakages in the USA annually, resulting in direct healthcare costs of over $\$ 17$ billion per year [3]. As reported by Strom et al.: "Osteoporosis causes more than 8.9 million fractures annually worldwide. Fracture rates are higher in the Western world than in other regions so that, despite the lower population, slightly more than one-third of all osteoporotic fractures occur in Europe" [1].

Approximately $21 \%$ of women aged $50-84$ years in the largest countries of the European Union (EU: Germany, Italy, Spain, UK) suffer from suffer from osteoporosis [4]. A more recent estimate of the healthcare costs associated with osteoporosis was $€ 29$ and $€ 38.7$ billion in the five large EU countries (France, Germany, Italy, Spain and UK) and in the $27 \mathrm{EU}$ countries, respectively. Hence the high societal and personal costs of osteoporosis pose challenges to public healthcare systems and physicians, particularly since most patients with fragility remain untreated. Estrogen deficiency seems to be the 
main factor of osteoporosis among menopausal women. According to the report of a World Health Organization study group [5] and other related studies [6,7], the risk factors for osteoporosis among women include the following:

- Advanced age ( $>50$ years)

- White or Asian ethnicity

- Genetic factors, such as a family history of osteoporosis

- Thin build or small stature (e.g., body weight less than $127 \mathrm{lb}$ )

- Amenorrhea

- Late menarche

- Early menopause

- Postmenopausal state

- Physical inactivity or immobilization

- Use of drugs: anticonvulsants, systemic steroids, thyroid supplements, heparin, chemotherapeutic agents, and insulin

- Alcohol and tobacco use

- Estrogen deficiency

- Calcium deficiency

- Dowager hump

Here we report our analysis of all articles entitled "Postmenopausal Osteoporosis" published in journals indexed in the Science Citation Index Expanded database (SCI-E) during the last decade (2001-2011). Our aim was to visualize the impact of leading countries in the field and to map the authorship network of authors contributing publications to Osteoporosis International between 2007 and 2011.

\section{Methods and materials}

All data were extracted from SCI-E. Two sets of data were analyzed. The first set included all papers entitled "Postmenopausal Osteoporosis" indexed in the SCI-E from 2001 to 2011. The title menu from field tags was used to limit the extraction of documents to papers entitled "Postmenopausal Osteoporosis." By restricting our search to titles we were able to identify the most relevant documents in desired subject areas. The keywords "Postmenopausal Osteoporosis" was selected from the lists of titles in the tags menu in SCI-E. This menu is based on the controlled vocabulary of Medical Subject Headings (MeSH).

The second set contained all papers published by Osteoporosis International that were indexed in the SCI-E between 2001 and 2011.

The Science of Science Tool was used to map the coauthorship networks of highly cited papers published by Osteoporosis International between 2007 and 2011 [8].

Data on the size of the population for the countries included in the analysis were extracted from the website of nation- online (http://www.nationsonline.org/ oneworld/populationby-country.htm) on 15 September 2013 [9].

\section{Results}

All papers indexed under the title of "Postmenopausal Osteoporosis" in the SCI-E during the study period (2001-2011) were extracted and analyzed. The extraction of data led to 2,056 papers entitled "Postmenopausal Osteoporosis" (Fig. 1).

As shown in Fig. 1, a total of 2,056 papers were published during the study period, with an average of 186.9 publications per year. The number of papers entitled "Postmenopausal Osteoporosis" in the SCI-E increased during the study period although there were some fluctuations. In terms of number of publications, the most prolific year was 2006 during which time 225 papers were published.

Table 1 shows the country of origin of papers entitled "Postmenopausal Osteoporosis" compiled in the SCI-E during the study period. The most prolific individual country in terms of number of publications was the USA, with $20 \%$ of all global publications entitled "Postmenopausal Osteoporosis" in SCI-E, followed by France (238 papers), England (198 papers), Italy (169), Switzerland (166), Belgium (161), Canada (159), Spain (154), Germany (149), and Turkey (122). In terms of regions, the majority of publications came from Western Europe and North America. Our comparison of the number of papers published by North American authors (the USA and Canada) and Western European authors revealed that the latter published approximately $75 \%$ more papers than North American authors. Most interesting was the finding that Iran, whose authors contributed 20 papers in the field, was ranked 30th among all 80 contributing countries. This placement was higher than that of Israel and India.

The last column (optimized rank) in Table 1 provides data on the number of published papers by each country divided by the number of inhabitants (in millions) of that same country.

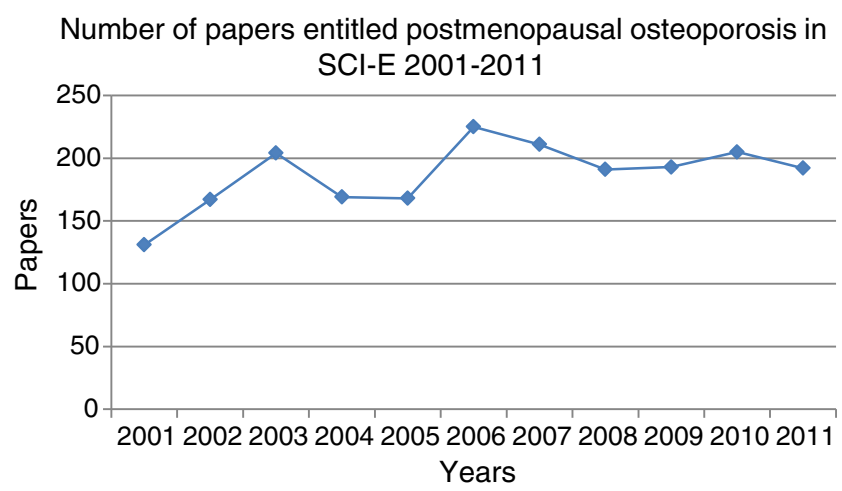

Fig. 1 Number of papers entitled "Postmenopausal Osteoporosis" in SCI-E 2001-2011 
Taking this optimized rank into account, the ten top productive countries (after reassessing the respective population size) were Switzerland, Denmark, Belgium, New Zealand, Slovenia, Scotland, Greece, Norway, Austria, and Canada. When we calculated the productivity of countries based on the number of inhabitants, we found that authors in Western Europe countries were more active than those in North America.

Figure 2 shows that about $50 \%$ of the total number of publications entitled "Postmenopausal Osteoporosis" were in

Table 1 Country of origin of papers entitled "Postmenopausal Osteoporosis" compiled in the SCI-E between 2001 and 2011

\begin{tabular}{|c|c|c|c|c|c|c|}
\hline Rank & Countries & Number of papers & Percentage & Population (in millions) & Number of papers/million population & Optimized rank \\
\hline 1 & USA & 696 & 20 & 316.555 & 2.2 & 21 \\
\hline 2 & France & 238 & 7 & 6.535 & 3.6 & 13 \\
\hline 3 & England & 198 & 6 & 53.012 & 3.7 & 12 \\
\hline 4 & Italy & 169 & 5 & 5.9464 & 2.8 & 17 \\
\hline 5 & Switzerland & 166 & 5 & 7.952 & 20.9 & 1 \\
\hline 6 & Belgium & 161 & 5 & 11.036 & 14.6 & 3 \\
\hline 7 & Canada & 159 & 5 & 35 & 4.5 & 10 \\
\hline 8 & Spain & 154 & 4 & 46.185 & 3.3 & 14 \\
\hline 9 & Germany & 149 & 4 & 80.5 & 1.9 & 23 \\
\hline 10 & Turkey & 122 & 4 & 74.724 & 1.6 & 25 \\
\hline 11 & Japan & 118 & 3 & 127.3 & 0.9 & 30 \\
\hline 12 & Denmark & 111 & 3 & 5.58 & 19.9 & 2 \\
\hline 13 & China & 65 & 2 & $1,347.35$ & 0 & 41 \\
\hline 14 & Poland & 61 & 2 & 38.501 & 1.6 & 26 \\
\hline 15 & Australia & 59 & 2 & 22.902 & 2.6 & 19 \\
\hline 16 & Argentina & 57 & 2 & 40.117 & 1.4 & 28 \\
\hline 17 & Greece & 56 & 2 & 10.787 & 5.2 & 7 \\
\hline 18 & Netherlands & 55 & 2 & 16.733 & 3.3 & 15 \\
\hline 19 & Austria & 43 & 1 & 8.45186 & 5.1 & 9 \\
\hline 20 & Sweden & 42 & 1 & 9.49 & 4.4 & 11 \\
\hline 21 & Brazil & 41 & 1 & 192.4 & 0.2 & 37 \\
\hline 22 & Romania & 40 & 1 & 19.043 & 2.1 & 22 \\
\hline 23 & New Zealand & 34 & 1 & 4.434 & 7.7 & 4 \\
\hline 24 & Czech Republic & 30 & 1 & 10.504 & 2.9 & 16 \\
\hline 25 & South Korea & 30 & 1 & 48.58 & 0.6 & 32 \\
\hline 26 & Scotland & 29 & 1 & 5.295 & 5.5 & 6 \\
\hline 27 & Ukraine & 27 & 1 & 45.644 & 0.6 & 33 \\
\hline 28 & Norway & 26 & 1 & 5.009 & 5.2 & 8 \\
\hline 29 & Hungary & 25 & 1 & 9.962 & 2.5 & 20 \\
\hline 30 & Iran & 20 & 1 & 76.8 & 0.3 & 35 \\
\hline 31 & Mexico & 20 & 1 & 112.336 & 0.2 & 38 \\
\hline 32 & Russia & 20 & 1 & 143.056 & 0.1 & 40 \\
\hline 33 & Taiwan & 19 & 1 & 23.29359 & 0.8 & 31 \\
\hline 34 & Slovenia & 15 & 0 & 2.07 & 7.2 & 5 \\
\hline 35 & South Africa & 15 & 0 & 50.586 & 0.3 & 36 \\
\hline 36 & Israel & 14 & 0 & 7.87 & 1.8 & 24 \\
\hline 37 & Croatia & 12 & 0 & 4.291 & 2.8 & 18 \\
\hline 38 & Bulgaria & 11 & 0 & 7.365 & 1.5 & 27 \\
\hline 39 & India & 11 & 0 & $1,210.57$ & 0 & 42 \\
\hline 40 & Portugal & 11 & 0 & 10.561 & 1 & 29 \\
\hline 41 & Saudi Arabia & 11 & 0 & 27.137 & 0.4 & 34 \\
\hline 42 & Thailand & 11 & 0 & 65.5 & 0.2 & 39 \\
\hline
\end{tabular}


Documents Type of papers entitled Postmenopausal osteoporosis in SCI-E 2001-2011

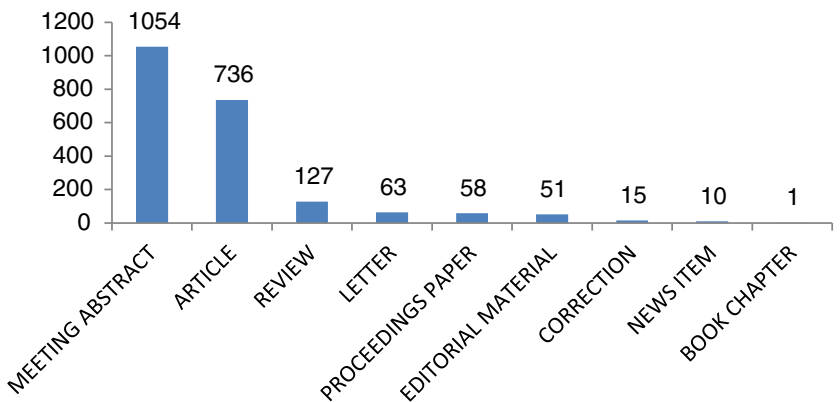

Fig. 2 Publication type of papers in the field of "Postmenopausal Osteoporosis" compiled in the SCI-E 2001-2011

the form of a meeting abstract. Full-length journal articles represented only $35 \%$ of the total number of publications.

Papers entitled "Postmenopausal Osteoporosis" compiled in the SCI-E during the study period were published in 329 different journals. Osteoporosis International published by far the majority of articles (22\%), followed by the journals Bone \& Mineral Research (13\%), Calcified Tissue International (4\%), Annals of the Rheumatic Disease (2\%), Arthritis and Rheumatism (2 \%), The North American Menopause Society (2 \%), Clinical Endocrinology Metabolism (2\%), Bone and Mineral Metabolism (2\%), and New England Journal of Medicine (1 \%) (Fig. 3).

The most prolific author was J.Y. Reginster from the University of Liege, Belgium, contributing a total of 84 papers, followed by P.D. Delmas of the University of Lyon, France (69 papers), C. Roux of the Paris Descartes University, France (56 papers), P.D. Miller of the Health Sciences Center, University of Colorado, USA (53 papers), C. Christiansen from the Clinical Research Center of CCBR-Ballerup, Denmark (51 papers), D. Felsenberg of the Universitatsklinikum Benjamin Franklin, Berlin, Germany (50 papers), R. Eastell of the University of Sheffield, Sheffield, UK (46 papers), J.D. Adachi from McMaster University, Hamilton, Ontario, Canada (45 papers), S. Boonen from the Center for Metabolic Bone Diseases, Catholic University of Leuven, Brussels, Belgium (45 papers), and S. Adami from Rheumatologic Rehabilitation,

Journals publishing papers entitled postmenopausal osteoprosis in SCI-E 2001-2011

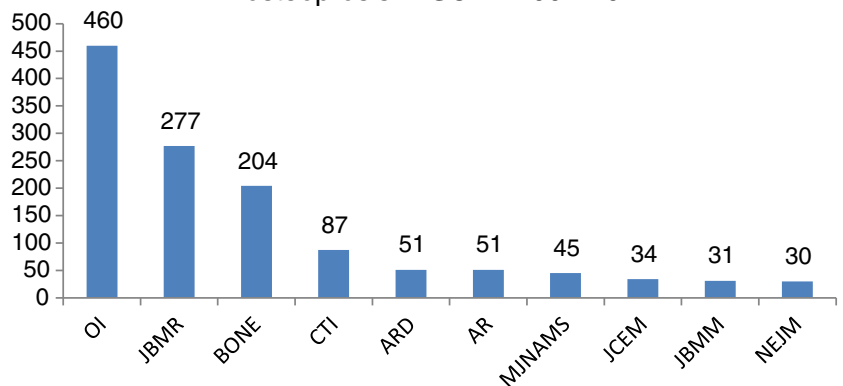

Fig. 3 Ten top productive journals publishing papers in the field of "Postmenopausal Osteoporosis" 2001-2011
Ten top productive authors contributing papers entitled as Postmenopausal osteoporosis in SCl-E 2001-2011

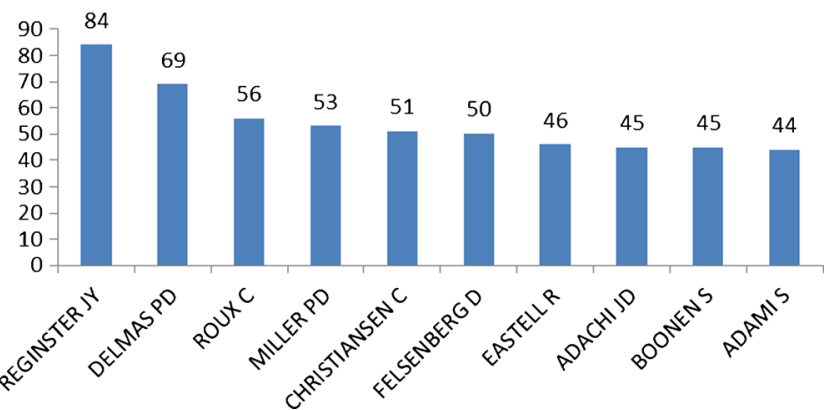

Fig. 4 Ten top productive authors contributing papers entitled as "Postmenopausal Osteoporosis" compiled in the SCI-E 2001-2011

University of Verona, Italy (44 papers). It is remarkable that of the top ten contributing authors, eight are from Western Europe and only two are from North America. This analysis was restricted to authors whose name appears on more than 40 papers entitled "Postmenopausal Osteoporosis" compiled in the SCI-E during the study period (Fig. 4).

A total of 9,024 papers published by Osteoporosis International were indexed in the SCI-E during the study period. Figure 5 shows that the most productive years were 2006 and 2011.

The majority of papers distributed by the Osteoporosis International originated from American authors followed by authors from England (1,271 papers) and France (738 papers). This analysis was restricted to the countries of the ten most productive authors who published in Osteoporosis International during the study period (Fig. 6).

The majority of papers published in Osteoporosis International were in the form of meeting abstracts (72\%) and full-length articles (23\%). It is notable that all papers published by this journal were in English and that only one paper was in Welsh (Table 2).

A total of 6,246 organizations contributed articles entitled "Postmenopausal Osteoporosis" compiled in the SCI-E during the study period. The analysis reported in Table 3 is restricted to the 20 top prolific organizations which published articles in

Number of papers indexed in SCI-E from Journal of Osteoprosis International 2001-2011

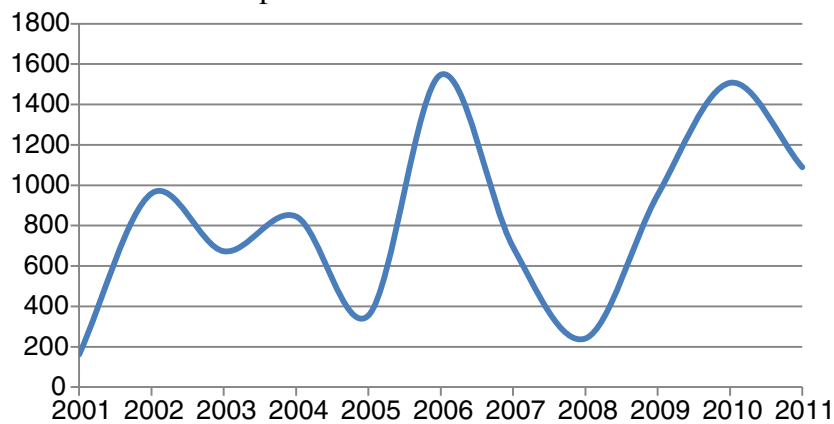

Fig. 5 Number of papers published by Osteoporosis International and indexed in SCI-E 2001-2011 
Osteoporosis International during the study period. The University of Sheffield, with its 261 published papers, was the most productive of these, followed by University of Liege (240 papers), and then by the University of California, San Francisco (190 papers). It is remarkable that among the 20 top productive organizations in the field, nine are from Western Europe.

A total number of 4,483 papers in different forms were published by Osteoporosis International during the period 2007-2011.

Figure 7 shows the co-authorship network of papers published by Osteoporosis International between 2007 and 2011. The map is restricted to those papers cited more than 100 times in the Web of Science during the period 2007-2011 and shows the strategic position of some authors in the network. Without the work of these authors the network would be divided into a number of small components. These strategic authors in the co-authorship network are called cut-points, and the link between them is called a bridge (in the case of missing these links, the network would embrace another isolated subnetworks; in other words, the network would experience a structural hole). These authors are:

1. J. A. Kanis, Medical School of Sheffield University, England, who has authored 68 papers cited 1,716 times in the WoS database.

2. C. Cooper, University of Oxford (NIHR Musculoskeletal Biomed Research Unit), England, has authored 105 papers which have been cited 960 times.

3. R. Rizzoli, Geneva University, Switzerland, has authored 62 papers which have been cited 510 times.

4. C. Roux, Paris Descartes University, France, has authored 58 papers which have been cited 204 times.

5. B. Dawson-Hughes, the Tuffs University. USA, has authored 23 papers which have been cited 640 times.

Productive countries countibuting papers in the journal of Osteoprosis International 2001-2011

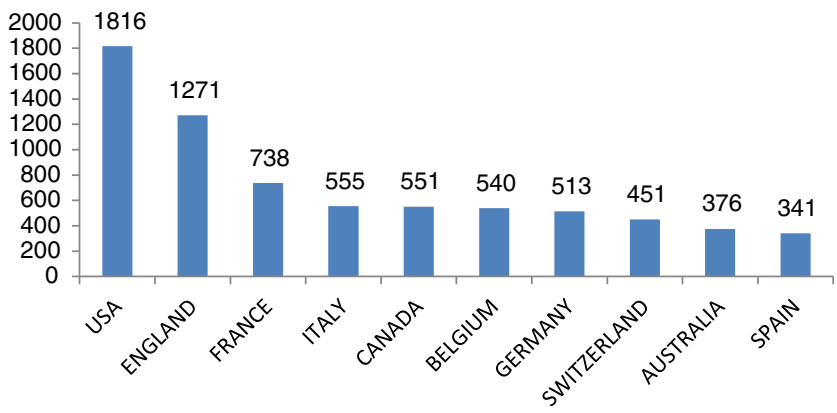

Fig. 6 Top ten productive countries contributing papers in Osteoporosis International 2001-2011
Table 2 Document type of publication from Osteoporosis International indexed in the SCI-E 2001-2011

\begin{tabular}{lrc}
\hline Document types & Number & Percentage \\
\hline Meeting abstract & 6,533 & 72 \\
Article & 2,088 & 23 \\
Review & 189 & 2 \\
Letter & 127 & 1 \\
Proceedings paper & 103 & 1 \\
Editorial material & 59 & 1 \\
Correction & 15 & 0 \\
Biographical item & 13 & 0 \\
Total & 9,127 & 100 \\
\hline
\end{tabular}

\section{Conclusion and discussion}

The main focus of our analysis was to evaluate the scientific literature on "Postmenopausal Osteoporosis" that had been indexed in the SCI-E from 2001 to 2011 . We found that during the study period a total of 2,056 articles entitled "Postmenopausal Osteoporosis" had been published during the study period and that a total of 2,228 institutes had published in the field of postmenopausal osteoporosis. There was a marked increase in the number of publications during the study period, although some fluctuations were observed. The highest

Table 3 Twenty top productive organizations contributing papers entitled "Postmenopausal Osteoporosis" compiled in the SCI-E 2001-2011

\begin{tabular}{llll}
\hline Rank & Organizations & Records & Percent \\
\hline 1 & University of Sheffield, UK & 261 & 2 \\
2 & University of Liege, Belgium & 240 & 2 \\
3 & University of California, San Francisco, USA & 190 & 2 \\
4 & McMaster University, Canada & 164 & 1 \\
5 & University of Melbourne, Australia & 128 & 1 \\
6 & University of Southampton, UK & 124 & 1 \\
7 & University of Toronto, Canada & 123 & 1 \\
8 & French National Institute of Health and & 121 & 1 \\
& Medical Research (INSERM), France & 115 & 1 \\
9 & Merck Co Inc, USA & 99 & 1 \\
10 & Hop Edouard Herriot, France & 97 & 1 \\
11 & University Hospitals, Geneva, Switzerland & 96 & 1 \\
12 & Eli Lilly Co, USA & 96 & 1 \\
13 & University of Cambridge, UK & 94 & 1 \\
14 & University Claude Bernard Lyon 1, France & 93 & 1 \\
15 & Procter Gamble Pharmaceuticals, USA & 92 & 1 \\
16 & Columbia University, USA & 91 & 1 \\
17 & University Hospitals, USA & 87 & 1 \\
18 & Harvard University, USA & 87 & 1 \\
19 & University of Manchester, UK & 82 & 1 \\
20 & Faculty of Medicine, Czech Republic & & \\
\hline & & &
\end{tabular}




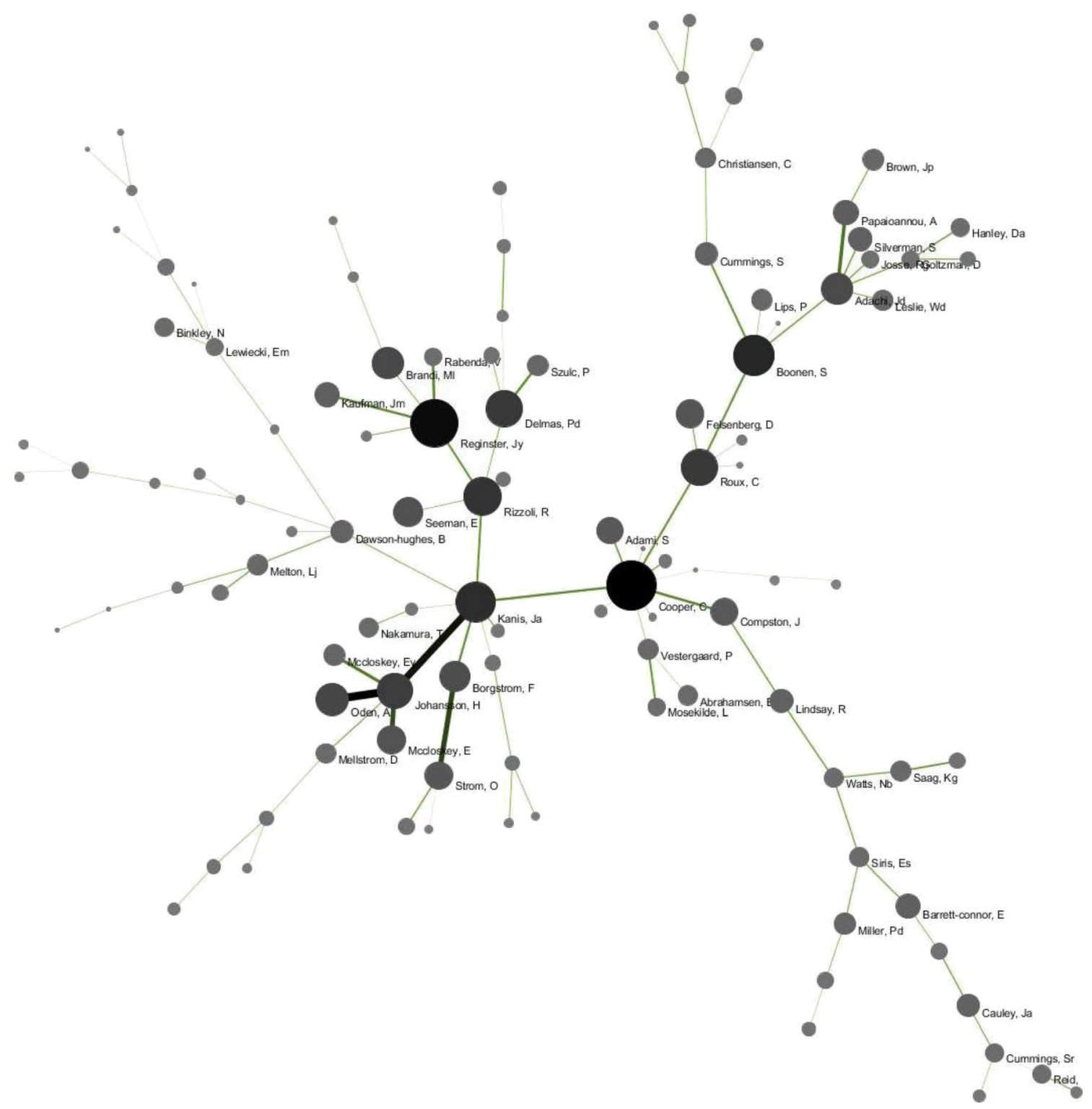

Fig. 7 Co-authorship network of papers distributed by Osteoporosis International 2007-2011

number of articles was published in 2006 (225 papers). During the study period Iranian scientists published over 20 papers in the field, exceeding the output from countries such as India and Israel. Full-length journal articles represented only $35 \%$ of the total number of publications, with the majority of publications in the field being in the form of meeting abstracts (approx. 50\% of total publications entitled "Postmenopausal Osteoporosis"). Based on these results, we suggest that this trend in publication type has occurred in accordance with the editorial policy of the related publication platforms, with their main focus on new subjects and themes in the field of osteoporosis. Nonetheless, it should be noted that new themes are first presented in conference meetings, with full publications often appearing later as journal articles. This clearly indicates that novelties in the field first appear as meeting abstracts.
A total of 329 journals published papers entitled "Postmenopausal Osteoporosis" throughout the study period. English was the dominant language of the published papers, which is not surprising since the editorial policy of the SCI-E is based upon the papers published in English [10]. Osteoporosis International published $22 \%$ of the total number publications; as such, it was the most prolific journal followed by the Journal of Bone and Mineral Research (13\%). In terms of authors, we found that J.Y. Reginster had the highest contribution rate (84 papers) followed by P.D. Delmas (69 papers), C. Roux (56 papers), P.D. Miller (53 papers), and C. Christiansen (51 papers).

Overall, a total number of 9,024 articles were published by Osteoporosis International during the study period, with 2006 and 2011 being the most prolific years in terms of number of 
published articles. The majority of papers published by Osteoporosis International originated from Western European authors. In terms of types of publications appearing in Osteoporosis International, $72 \%$ were in the form of meeting abstracts and only $23 \%$ were in the form of full-length journal articles. It is notable that all papers published in this journal were in English, while only one paper was in the Welsh language. With a total of 261 published papers, the University of Sheffield was the most productive institute contributing papers to Osteoporosis International, followed by the University of Liege in Belgium (240 papers) and the University of California, San Francisco (UCSF) (190 papers).

In total, 19,444 authors published their studies in Osteoporosis International through the study period 2001-2011. J.Y. Reginster from the University of Liege, Belgium, with 272 papers published in Osteoporosis International, was the most productive scientist, followed by C. Cooper C (185 papers) and P.D. Delmas from the University of Lyon, France (159 papers), and J.A. Kanis from the University of Sheffield, England (141 papers).

A total number 4,483 papers in different formats were published in Osteoporosis International during the period 2007-2011. Our mapping of the co-authorship network of highly cited papers published by Osteoporosis International indicates the strategic position of the authors from four developed countries (i.e., England, France, Switzerland, and USA). The most strategically positioned authors in the network (cutpoints) were J.A. Kanis (University of Sheffield), who has authored 68 papers with a total number of citations of 1,716 in the WoS during the period 2007-2011, C. Cooper (University of Oxford), who has authored 105 papers with a total number of citations of 960 . Without the work of these authors, the network would be divided in two smaller components.

It should be pointed out that although the USA was the most prolific individual country in terms of number of publications, far more articles were published from Western European authors. Our comparison of papers published by authors from the USA/Canada (North America) and those published by authors from Western Europe revealed that the latter had a $75 \%$ higher publication rate than North American authors. The network of co-authorship emphasized the strategic positions of highly cited authors from Western Europe. Of the ten top authors contributing to the majority of papers, eight were from Western Europe. Consequently, Western Europe had a more important impact than North America during the study period.

Acknowledgments Authors would like to thank Prof. Y. Omidi (Tabriz University of Medical Sciences, Iran) for his technical advice.

Conflicts of interest None.

\section{References}

1. Strom O, Borgstrom F, Kanis JA, Compston J, Cooper C, McCloskey EV, Jonsson B (2011) Osteoporosis: burden, health care provision and opportunities in the EU: a report prepared in collaboration with the International Osteoporosis Foundation (IOF) and the European Federation of Pharmaceutical Industry Associations (EFPIA). Arch Osteoporos 6(1-2):59-155. doi:10.1007/s11657-011-0060-1

2. http://www.betterhealth.vic.gov.au/bhcv2/bhcarticles.nsf/pages/ Menopause_and_osteoporosis. Accessed 7 August 2013

3. http://www.austinthyroid.com/osteoporosis.htm. Accessed 07 August 2013

4. Kanis JA, Burlet N, Cooper C et al (2008) European guidance for the diagnosis and management of osteoporosis in postmenopausal women. Osteoporos Int 19:399-428. doi:10.1007/s00198-008-0560-Z

5. Report of a WHO Study Group (1994) Assessment of fracture risk and its application to screening for postmenopausal osteoporosis. World Health Organ Tech Rep Ser 843:1-129

6. Lyles KW, Schenck AP, Colon-Emeric CS (2008) Hip and other osteoporotic fractures increase the risk of subsequent fractures in nursing home residents. Osteoporos Int 19(8):1225-1233. doi:10. 1007/s00198-008-0569-3

7. Fink HA, Kuskowski MA, Taylor BC, Schousboe JT, Orwoll ES, Ensrud KE (2008) Association of Parkinson's disease with accelerated bone loss, fractures and mortality in older men: the Osteoporotic Fractures in Men (MrOS) study. Osteoporos Int 19(9):1277-1282. doi:10.1007/s00198-008-0584-4

8. $\mathrm{Sci}^{2}$ Team (2009) Science of Science ( $\left.\mathrm{Sci}^{2}\right)$ Tool. Indiana University and SciTech Strategies. http://sci2.cns.iu.edu

9. Nation-online (http://www.nationsonline.org/oneworld/populationby-country.htm). Accessed 07 August 2013

10. Biglu MH, Umstatter W (2007) The editorial policy of languages is being changed in Medline. Acimed 16:3 